\title{
Влияние разориентации подложки на состав, структурные и фотолюминесцентные свойства эпитаксиальных слоев, выращенных на GaAs(100)
}

\author{
() П.В. Середин ${ }^{1}$, А.С. Леньшин ${ }^{1}$, А.В. Федюкин ${ }^{1}$, И.Н. Арсентьев ${ }^{2}$, А.В. Жаботинский ${ }^{2}$, \\ Д.Н. Николаев ${ }^{2}$, Harald Leiste ${ }^{3}$, Monika Rinke ${ }^{3}$
}

${ }^{1}$ Воронежский государственный университет, 39006 Воронеж, Россия

${ }^{2}$ Физико-технический институт им. А.Ф. Иоффре Российской академии наук, 194021 Санкт-Петербург, Россия

${ }^{3}$ Karlsruhe Nano Micro Facility,

76344 Eggenstein-Leopoldshafen, Germany

E-mail: paul@phys.vsu.ru; arsentyev@mail.ioffe.ru

(Получена 21 фревраля 2017 г. Принята к печати 3 марта 2017 г.)

Исследовано влияние степени разориентации подложки GaAs, а также ее обработки на структурные и оптические характеристики гомоэпитаксиальных структур GaAs/GaAs(100), выращенных методом MOCVD. На основе данных комплекса структурных и спектроскопических методов показано, что увеличение степени отклонения подложки от точной ориентации до $4^{\circ}$ к [110] приводит к ступенчатому росту пленки GaAs на первоначальном этапе, а дальнейшее увеличение величины разориентации до $10^{\circ} \mathrm{\kappa}[110]$ приводит к росту числа дефектов в структуре эпитаксиальной пленки. При этом образцы гомоэпитаксиальных структур, выращенные методом MOCVD на подложках GaAs (100) с разориентацией 4 ${ }^{\circ}$ [110], обладают максимальным выходом фотолюминесценции, превосходящим примерно на $15 \%$ аналогичную величину для структур, выращенных на точно ориентированной подложке $\mathrm{GaAs}(100)$. Предварительная полировки подложки GaAs (удаление окисного слоя) также приводит к возрастанию эмиссии фотолюминесценции по сравнению с неполированной подложкой того же типа, а для образцов, выращенных на подложках с разориентацией $4^{\circ}$ такое увеличение выхода фотолюминесценции составляет величину 30\%.

DOI: $10.21883 /$ FTP.2018.01.45329.8565

\section{1. Введение}

Полупроводники группы GaAs более сорока лет являются основным материалом, используемым для производства традиционных устройств оптоэлектроники: солнечных батарей, фотоприемников, светодиодов и инжекционных лазеров $[1,2]$. Однако в условиях миниатюризации конечных устройств на основе $\mathrm{A}^{\mathrm{III}} \mathrm{B}^{\mathrm{V}}$ производство оптоэлектронной компонентной базы требует повышения функциональных свойств классических материалов [3], в том числе и для дальнейшей оптоволоконной интеграции полупроводников $\mathrm{GaAs}$ с различными подложками, в том числе и с кремниевой схемой обработки сигналов. Поэтому поиск технологических приемов получения эпитаксиальных гетероструктур на основе $\mathrm{A}^{\mathrm{III}} \mathrm{B}^{\mathrm{V}}$ с улучшенными функциональными свойствами продолжается [4,5].

Одним из подходов, широко используемых при производстве оптоэлектронных компонент и активно развиваемых в последнее время, является рост на подложках с разориентацией [6-8].

Как показывает эксперимент, проблемы качества эпитаксиальных слоев и их морфологии [9] могут быть решены с использованием такого подхода. Так, в случае интеграции GaAs с кремнием [10], применение подложек $\mathrm{Si}$, отклоненных от сингулярной плоскости (001) на $4-6^{\circ}$, позволяет избавиться от образования анти- фазных доменов большой плотности, мешающих интеграции. Что касается роста самосогласованных по параметру решетки гетероструктур $\mathrm{AlGaAs} / \mathrm{GaAs}$ типа, то, как уже было показано авторами работы [11] использование подложек $\operatorname{GaAs}(111) \mathrm{A}$ с разориентацией к направлению [100] позволило не только контролировать качество поверхности, но и управлять оптическими свойствами [12,13], распределением и размерами квантово-размерных неоднородностей в эпитаксиальной пленке [14], а также типом и концентрацией введенной примеси [15-17].

Также в ряде работ было показано, что с ростом величины разориентации подложки при росте гетероструктур с большой разницей между параметрами гетеропары возможно усиление квантового выхода и сужение полосы фотолюминесценции, что является показателем улучшения оптических и структурных свойств [18]. Однако даже в хорошо согласованных по параметру решетки гетероструктурах [8], выращенных на подложках $\operatorname{GaAs}(100)$ с разориентацией $<1^{\circ}$, и в гетеростуктурах с квантовыми ямами [7], выращенных на подложках $\operatorname{GaAs}(100)$ с высоким отклонением от направления [110], использование разориентированных подложек позволило эффективно управлять морфологией поверхности и оптическими характеристиками образцов.

Однако мы не нашли в литературе информации о влиянии разориентации подложки $\mathrm{GaAs}(100)$ на струк- 
Описание исследованных образцов гомоэпитаксиальных структур $\mathrm{GaAs} / \mathrm{GaAs}(100)$

\begin{tabular}{|c|c|c|c|c|c|c|c|c|}
\hline Образцы & S1_0 & S1_2 & S1_4 & S1_10 & S2_2_np & $\mathrm{S} 2 \_2 \_p$ & S2_4_np & $\mathrm{S} 2 \_4 \_p$ \\
\hline Подложка & \multicolumn{4}{|c|}{$\mathrm{GaAs}(100)$} & \multicolumn{4}{|c|}{$\operatorname{GaAs}(100)$} \\
\hline Разориентация & $0^{\circ}$ & $2^{\circ}$ к $[110]$ & $4^{\circ} \mathrm{K}[110]$ & $10^{\circ}$ к $[110]$ & $2^{\circ}$ к $[110]$ & $2^{\circ}$ к $[110]$ & $4^{\circ}$ к $[110]$ & $10^{\circ}$ к $[110]$ \\
\hline Полировка & + & + & + & + & - & + & - & + \\
\hline
\end{tabular}

турные и фотолюминесцентные свойства гетероструктур с полностью согласованными параметрами решетки у гетеропары, а также на состав растущих слоев при одинаковых условиях роста.

В данной работе приведены результаты исследований вышеописанной проблемы.

\section{2. Объекты и методы исследования}

В работе исследовались две серии эпитаксиальных гетероструктур, выращенных методом MOCVD на подложках $\operatorname{GaAs}(100)$ с различной ориентацией.

Тестируемые образцы представляют собой гомоэпитаксиальные структуры GaAs/GaAs(100) (серия S1 и S2). Структуры выращивались методом MOCVD на установке „ЕMCORE GS 3/100“ в вертикальном реактоpe c высокой скоростью вращения подложкодержателя. Температура подложкодержателя варьировалась в пределах $650-750^{\circ} \mathrm{C}$, давление в реакторе $77 \mathrm{Topp}$, скорость вращения подложкодержателя 1000 об/мин. В качестве исходных реагентов использовались триметилгаллия $\mathrm{Ga}\left(\mathrm{CH}_{3}\right)_{3}$, триметилалюминия $\mathrm{Al}\left(\mathrm{CH}_{3}\right)_{3}$ и арсин $\mathrm{AsH}_{3}$. Описания образцов серии S1 и S2 приведены в таблице.

Серии образцов S1 и S2 были выращены для определения структурного качества гомоэпитаксиальных слоев и величины квантового выхода фотолюминесценции в зависимости от величины разориентации подложки, а также ее полировки. Каждая серия гетероструктур была выращена в одном технологическом процессе.

Структурное качество образцов и определение параметров решеток твердых растворов проводили методом рентгеновской дифракции на дифрактометре Seifert 3003 HR с четырехкружным гониометром и монохроматизированным излучением меди с длиной волны $\mathrm{Cu} K_{\alpha 1}=1.5405 \AA$.

Спектры рамановского рассеяния были получены на спектрометре Renishaw 1000, оснащенным микроскопом с объективом $50 \times$ NPlan. Для возбуждения спектров использовался аргоновый лазер с длиной волны 514.5 нм. Мощность лазерного пучка на поверхности образца не превышала $3 \mu \mathrm{BT}$.

Спектры фотолюминесценции гетероструктур были получены при комнатной температуре по стандартной методике с помощью монохроматора TRIAX550. Для возбуждения спектров ФЛ использовали аргоновый лазер с длиной волны 514.5 нм. Для фокусировки лазер- ного луча на поверхность образца был использован объектив $10 \times$. Сигнал регистрировался с помощью охлаждаемого жидким азотом CCD-детектора.

\section{3. Экспериментальные результаты и их обсуждение}

Структурное качество образцов гомоэпитаксиальных структур, а также гетероструктур с квантовыми ямами выращенных на подложках с различной степенью разориентации, было выполнено с применением высокоразрешающей рентгеновской дифракции. Такие исследования были проведены нами с использованием карт обратного $q$-пространства образцов, поскольку именно они позволяют получать прямую информацию о рассогласовании параметров кристаллических решеток эпитаксиальной пленки и подложки, разориентации или релаксации слоя, плотности дислокаций в нем, его мозаичности или кривизне $[19,20]$.

На рис. 1 представлены карты распределения интенсивности дифрагированного излучения в $q$-пространстве вокруг симметричного в (400) и асимметричного (511) узлов для серии образцов $\mathrm{S} 1$.

Анализ симметричного и асимметричного сканов обратного пространства (рис. 1) показывает, что на каждой карте присутствует лишь один узел, что указывает на когерентный рост эпитаксиальных пленок GaAs на подложках $\mathrm{GaAs}(100)$ с различной степенью разориентации относительно направления [110].

Однако, как хорошо видно из рис. 1 , положение и форма узлов в обратном пространстве как для симметричного, так и для асимметричного отражения изменяются. На рисунке показано размазывание узлов на картах (511) в направлениях $q^{\|}$и $q^{\perp}$ для образцов с рассогласованием 2 и $4^{\circ}$, а для образца S1_10, выращенного на подложке с рассогласованием $10^{\circ}$, лишь в направлении $q^{\perp}$. В первом случае такое изменение формы узла, характерное в большей степени при понижении углового разрешения при регистрации карты обратного пространства, свидетельствует о ступенчатом росте пленки GaAs и появлении напряжений в эпитаксиальном слое в плоскости роста. Что касается образца S1_10, то изменение формы узла показывает увеличение дефектности структуры эпитаксиальной пленки.

На картах (511) для образцов S1_2 и S1_4 присутствуют дополнительные сателлиты (рефлексы). Интенсивность и форма этих отражений указывают на тот факт, 

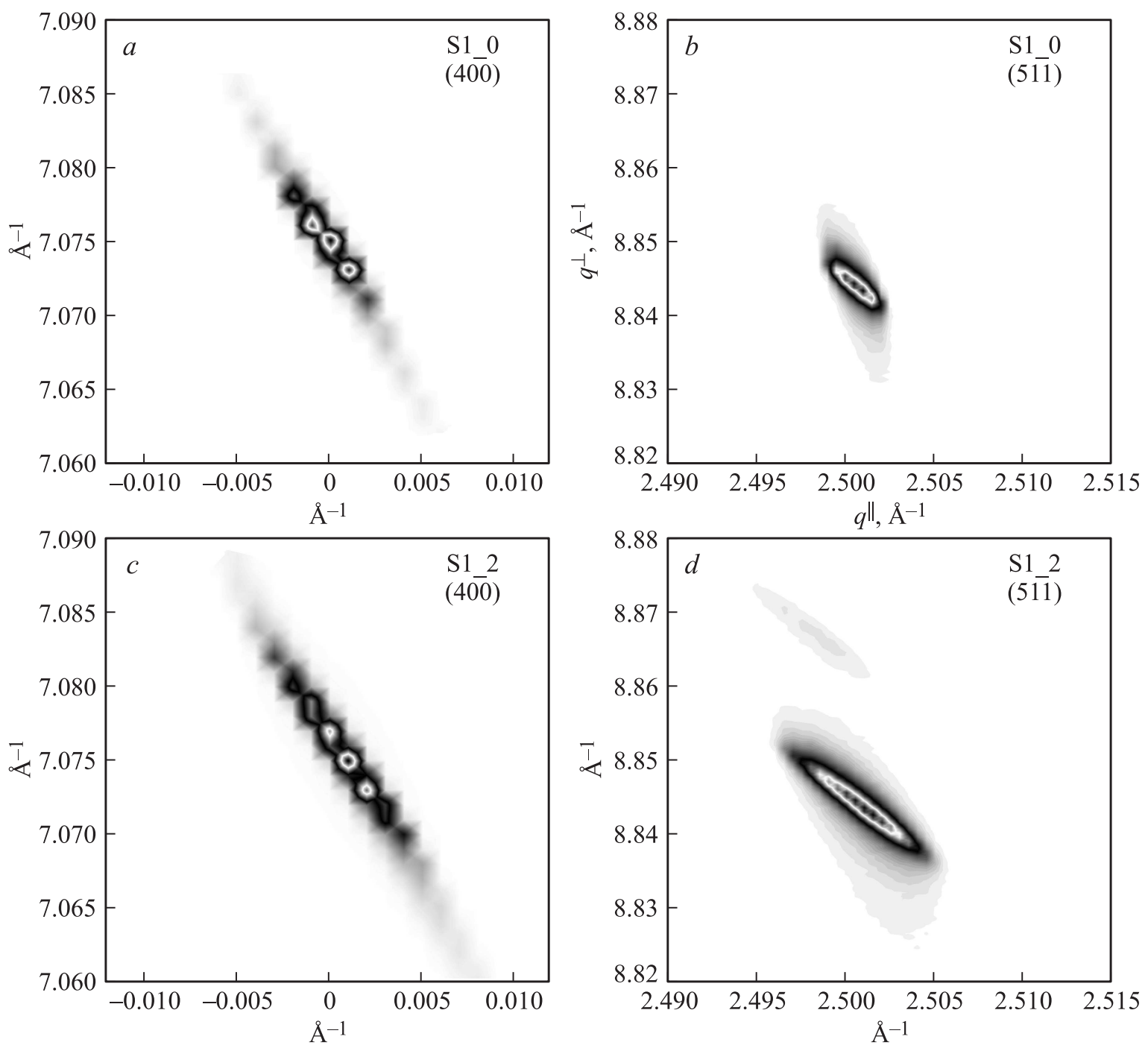

Рис. 1. Карты обратного $q$-пространства, полученные вокруг узлов (400) (слева) и (511) (справа) образцов гомоэпитаксиальных структур $\mathrm{GaAs} / \mathrm{GaAs}(100)$, выращенных на подложках с разной ориентацией: $a, b-$ образец $\mathrm{S} 1 \_0$ (разориентация $0^{\circ}$ ); $c, d-$ образец S1_2 (разориентация $2^{\circ}$ к [110]); $e, f$ - образец S1_4 (разориентация $4^{\circ}$ к [110]); $g, h$ - образец S1_10 (разориентация 10 К $[110])$.

что они получены от весьма тонкого слоя (на 2 порядка меньше толщины эпитаксиальной пленки) с параметром решетки меньшим, чем у GaAs.

Кроме того было отмечено, что с ростом величины отклонения изменится наклон узлов по отношению к осям $q^{\perp}$ и $q^{\|}$. При этом расчет угла наклона показал, что в случае точно ориентированной подложки угол наклона узла на картах (400) и (511) совпадает.

Для получения дополнительной информации о влиянии разориентации подложки на структурные свойства полученных эпитаксиальных пленок был использован метод рамановской спектроскопии [20]. Этот метод позволяет исследовать кристаллическую структуру и ее качество.

Глубина проникновения лазерного излучения с длиной волны $\lambda=532$ нм, а следовательно, и эффективная глубина анализа при рамановском рассеянии может быть определена из соотношения $\lambda / 2 \pi k$, где $k-$ коэффициент экстинкции. Соответственно в случае анализа системы GaAs такая глубина составит приблизительно $\sim 200$ нм.

Согласно правилам отбора, полученным из анализа тензоров рамановского рассеяния [21] для кристаллов с алмазной структурой при обратном рассеянии от (100) поверхности, могут наблюдаться только LO фононы, а появление ТО фононов запрещено.

На рис. 2 приведены спектры рамановского рассеяния в геометрии $x(y, z) \bar{x}$ для образцов гомоэпитаксиальных структур, выращенных на подложках $\mathrm{GaAs}(100)$ с различной степенью разориентации (серия S1).

Как видно из полученных результатов (см. рис. 2), в спектрах всех образцов основной высокоинтенсивной модой является продольное колебание $\mathrm{LO}_{\mathrm{Ga}-\mathrm{As}}$, характерное для образов данного типа. Интенсивность 

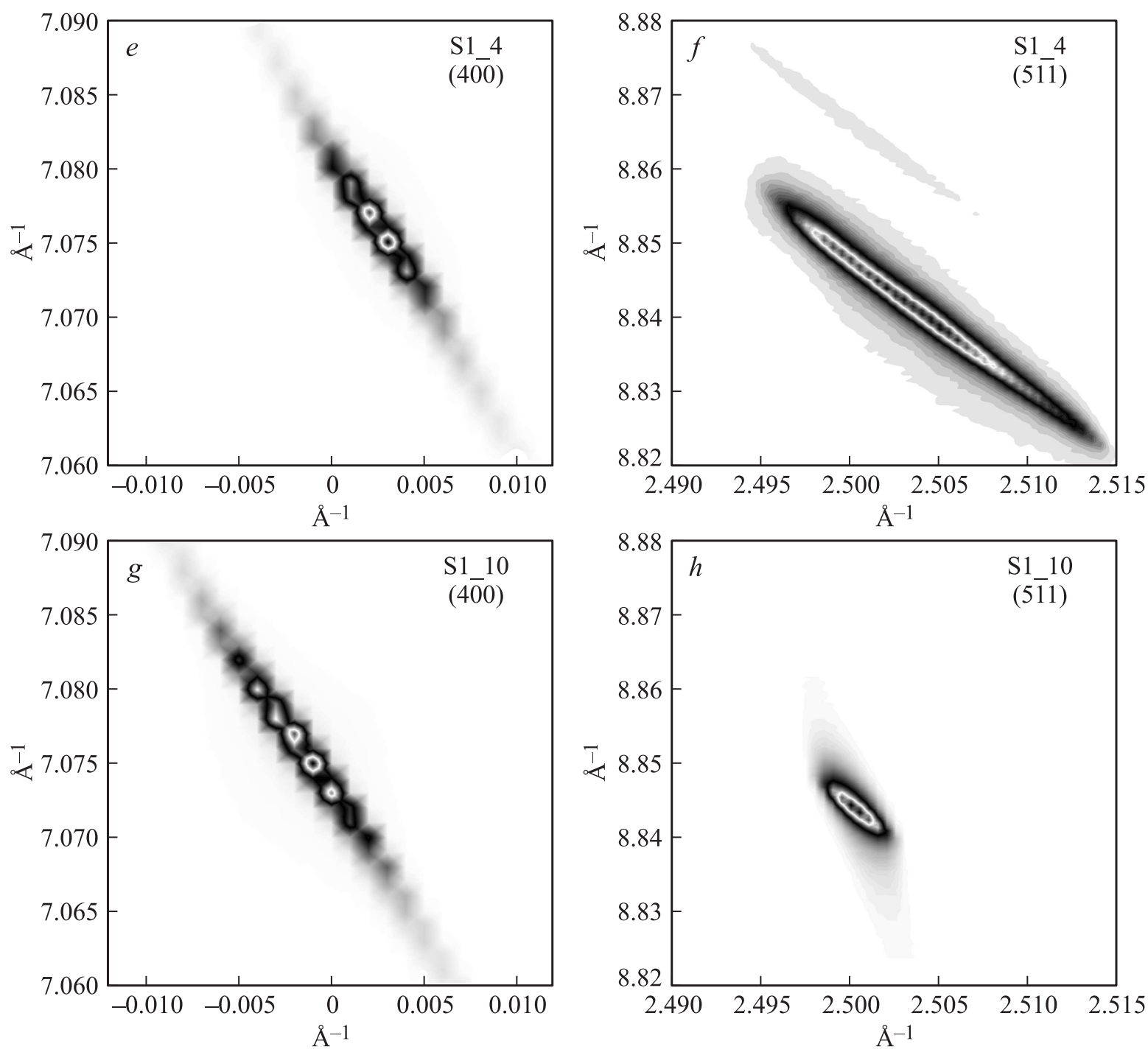

Рис. 1 (продолжсене).

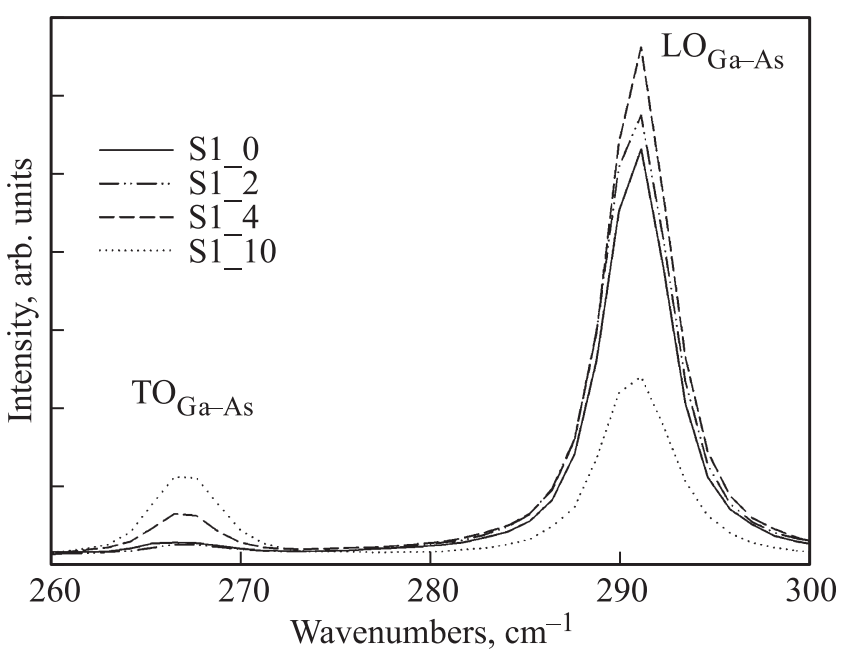

Рис. 2. Спектры рамановского рассеяния гомоэпитаксиальных структур GaAs/GaAs(100), выращенных на подложках с разной ориентацией. сигнала рамановского рассеяния максимальна для образца S1_4, выращенного на подложке с отклонением $4^{\circ}$ от [110]. Следует отметить, что с ростом величины разориентации монокристаллической подложки GaAs от направления (100) в рамановском спектре эпитаксиальной пленки GaAs начинает появляться дополнительная мода. Это колебание является запрещенным для данной геометрии эксперимента фононом $\mathrm{TO}_{\mathrm{Ga}-\mathrm{As}}$, появление которого может быть обусловлено нарушением симметрии кристаллической решетки в эпитаксиальной пленке из-за возникновения и возрастания числа структурных дефектов при росте на подложке с отклонением от плоскости (100). Анализ полученных результатов также показывает (см. рис. 2), что с ростом величины разориентации подложки GaAs от плоскости (100) интенсивность колебания $\mathrm{TO}_{\mathrm{Ga}-\mathrm{As}}$ возрастает, а следовательно, уменьшается структурное качество кристалла.

Экспериментальные данные, полученные методом рамановской спектроскопии, хорошо согласуются с ре- 


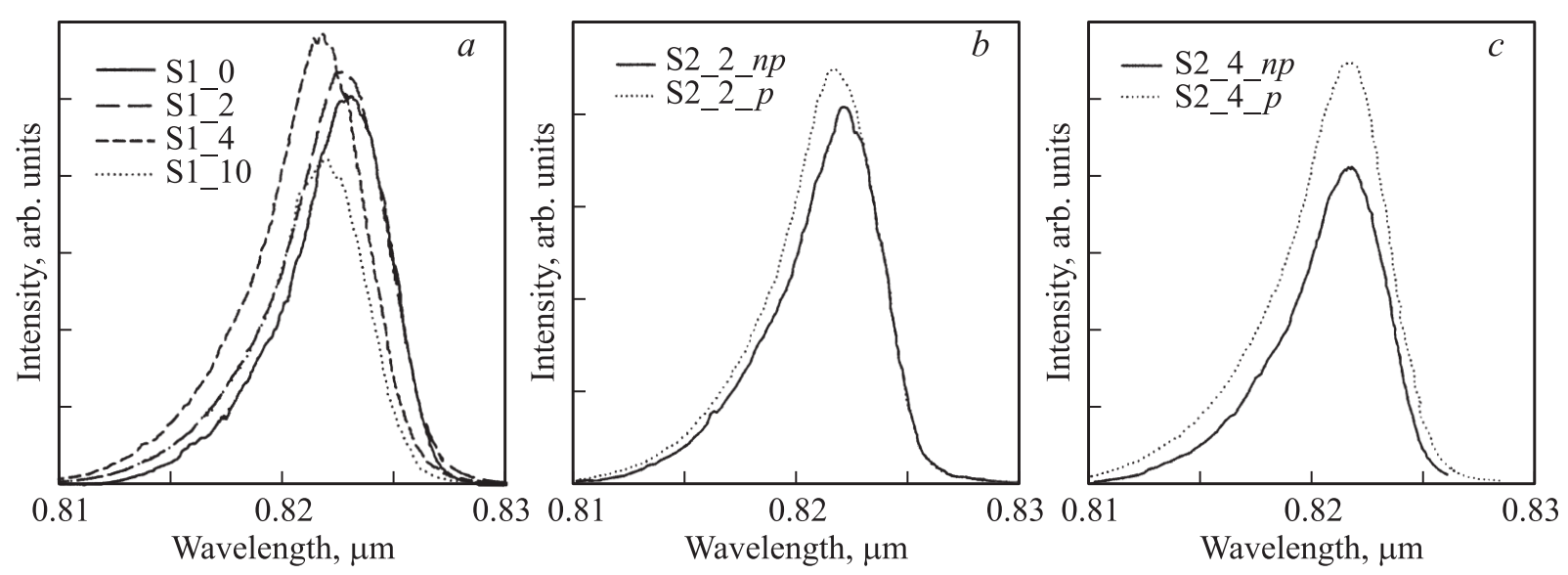

Рис. 3. Спектры фотолюминесценции гомоэпитаксиальных структур $\mathrm{GaAs} / \mathrm{GaAs}(100)$, полученные при комнатной температуре: $a$ - спектры образцов серии S1, выращенных на подложках с разной ориентацией; $b$ - спектры образцов серии S2 (разориентация $2^{\circ}$ к $[110]$ ), выращенных на подложках до и после полировки; $c$ - спектры образцов серии S2 (разориентация $4^{\circ}$ к [110]), выращенных на подложках до и после полировки.

зультатами высокоразрешающей рентгеновской дифрактометрии о структурном качестве образцов, выращенных на подложках $\mathrm{GaAs}(100)$ с разной степенью разориентации.

Изменение стехиометрии в эпитаксиальном слое неизменно должно отразиться на характере и величине квантового выхода фотолюминесценции от образца $[8,18,22-24]$, т. е. на энергетических характеристиках арсенида галлия, выращенного на подложке $\mathrm{GaAs}(100)$ с отклонением от плоскости (100). Для проверки этого утверждения нами были получены спектры фотолюминесценции при температуре жидкого азота. Спектры образцов, выращенных на подложках с разной степенью разориентации подложки (серия S1), приведены на рис. 3, $a$ без нормировки. Из полученных результатов видно, что в спектрах гомоэпитаксиальных структур $\mathrm{GaAs} / \mathrm{GaAs}(100)$ присутствует лишь один максимум с энергией $\sim 1.50$ эВ, представляющий собой эмиссию от гомоэпитаксиального слоя. Хорошо видно, что с ростом величины разориентации подложки $\mathrm{GaAs}(100)$ интенсивность выхода фотолюминесценции возрастает и достигает своего максимума у образца S1_4, выращенного на GaAs с рассогласованием $4^{\circ}$. В случае данного образца эффективность фотолюминесценции примерно на $15 \%$ выше, чем у структуры, выращенной на точно ориентированной подложке $\mathrm{GaAs}(100)$.

Следует также отметить, что рост на разориентированной подложке не только приводит к изменению эффективности фотолюминесценции, но и отражается на энергетическом положении максимума эмиссии (см. рис. $3, a)$ [25].

Однако, как показывает практика, на конечную величину эффективности фотолюминесценции гомоэпитаксиальной структуры $\mathrm{GaAs} / \mathrm{GaAs}(100)$ оказывает влияние не только величина разориентации использованной подложки GaAs, но и ее предварительная обработка. На рис. $3, b$ и $c$ представлены результаты исследования влияния предварительной полировки разориентированных подложек GaAs (от окисного слоя) на их фотолюминесцентные свойства. Как хорошо видно из полученных результатов, интенсивность эмиссии гетероструктуры образцов серии S2, выращенных в одном цикле на подложках с разориентацией 2 и $4^{\circ}$, весьма сильно зависит от предварительной обработки GaAs. Если в случае образцов, выращенных на подложках $\mathrm{GaAs}(100)$ с разориентацией $2^{\circ}$, после полировки подложки происходит возрастание эмиссии на величину $\sim 10 \%$ по сравнению с неполированной подложкой того же типа, то для образцов, выращенных на подложках с разориентацией $4^{\circ}$, характерно увеличение эффективности фотолюминесценции более чем на $30 \%$.

\section{4. Заключение}

Анализируя результаты исследований, полученные комплексом структурных и спектроскопических методов, о влиянии степени разориентации подложки GaAs, а также ее обработке на рост и характеристики гомоэпитаксиальных структур $\mathrm{GaAs} / \mathrm{GaAs}(100)$, выращенных методом MOCVD, можно сделать следующие заключения.

Увеличение степени отклонения подложки от точной ориентации до $4^{\circ}$ к [110] приводит к ступенчатому росту пленки GaAs на первоначальном этапе и появлению напряжений в эпитаксиальном слое в плоскости роста. Дальнейшее увеличение величины разориентации (до $10^{\circ}$ к [110]) ведет к росту числа структурных дефектов в эпитаксиальной пленке. Эти данные о структурном качестве образцов, выращенных на подложках $\mathrm{GaAs}(100)$ с разной степенью рассогласования, подтверждают результаты рамановской спектроскопии. Анализ фононного спектра подтверждает, что нарушение симметрии кристаллической решетки в эпитаксиальной 
пленке происходит за счет увеличения числа дефектов в эпитаксиальной пленке при росте на подложке $\mathrm{GaAs}$ c разориентацией.

Однако использование подложек $\mathrm{GaAs}(100)$ с разориентацией $4^{\circ}$ к [110] демонстрирует максимальную интенсивность продольного оптического фонона $\mathrm{LO}_{\mathrm{Ga}-\mathrm{As}}$ в рамановском спектре и минимальное соотношение интенсивностей продольного и поперечного оптических фононов, характеризующее структурное качество пленки GaAs. Помимо этого, образцы гомоэпитаксиальных структур, выращенные методом MOCVD на подложках $\operatorname{GaAs}(100)$ с разориентацией $4^{\circ}$ к [110], обладают максимальной эффективностью фотолюминесценции, превосходящей примерно на $15 \%$ аналогичную величину для структур, выращенных на точно ориентированной подложке $\mathrm{GaAs}(100)$. Предварительная полировки подложки GaAs также приводит к возрастанию эмиссии по сравнению с неполированной подложкой того же типа, а для образцов, выращенных на подложках с разориентацией $4^{\circ}$ такое увеличение выхода фотолюминесценции составляет величину 30\%.

Полученные результаты показывают, что выбор степени разориентации подложки GaAs, eе предварительная обработка, так же как и выбор условий роста гетероструктур, определяют их дальнейшие оптические/эмиссионные свойства и дают большую гибкость для дальнейших технологических разработок в области конструирования оптоэлектронных компонент.

Работа в части создания и фундаментальных исследований эпитаксиальных гетероструктур с высокими функциональными свойствами выполнена при поддержке гранта президента РФ МД-188.2017.2.

Работа в части разработки технологии получения и исследования эпитаксиальных гетероструктур выполнена в соответствии с государственным заданием ФТИ им. А.Ф. Иоффе.

Работа в части диагностики гетероструктур выполнена при поддержке гранта № 11.4718.2017/БЧ Министерством образования и науки России в рамках государственного задания вузам в сфере научной деятельности на 2017-2019 гг.

We acknowledge the Karlsruhe Nano Micro Facility (KNMF, www.kit.edu/knmf) of the Forschungszentrum Karlsruhe for provision of access to instruments at their laboratories.

Авторы выражают благодарность В.А. Капитонову за помощь в проведении измерений спектров фотолюминесценции образцов.

\section{Список литературы}

[1] S. Moon, K. Kim, Y. Kim, J. Heo, J. Lee. Sci. Rept., 6, 30107 (2016).

[2] P.V. Seredin, A.V. Glotov, E.P. Domashevskaya, I.N. Arsentyev, D.A. Vinokurov, I.S. Tarasov. Appl. Surf. Sci., 267, 181 (2013).
[3] P.V. Seredin, A.V. Glotov, V.E. Ternovaya, E.P. Domashevskaya, I.N. Arsentyev, D.A. Vinokurov, A.L. Stankevich, I.S. Tarasov. Semiconductors, 45, 481 (2011).

[4] P.V. Seredin, A.V. Glotov, V.E. Ternovaya, E.P. Domashevskaya, I.N. Arsentyev, L.S. Vavilova, I.S. Tarasov. Semiconductors, 45, 1433 (2011).

[5] P.V. Seredin, A.V. Glotov, E.P. Domashevskaya, I.N. Arsentyev, D.A. Vinokurov, I.S. Tarasov. J. Phys. B: Condens. Matter, 405, 4607 (2010).

[6] R. Boussaha, H. Fitouri, A. Rebey, B.E. Jani. Appl. Surf. Sci., 291, 40 (2014).

[7] H. Dong, J. Sun, S. Ma, J. Liang, T. Lu, X. Liu, B. Xu. Nanoscale, 8, 6043 (2016).

[8] E. Pelucchi, N. Moret, B. Dwir, D.Y. Oberli, A. Rudra, N. Gogneau, A. Kumar, E. Kapon, E. Levy, A. Palevski. J. Appl. Phys., 99, 093515 (2006).

[9] E.S. Johnson, G.E. Legg. J. Cryst. Growth, 88, 53 (1988).

[10] H.-S. Kim, Y. Kim, M.-S. Kim, S.-K. Min. J. Cryst. Growth, 92, 507 (1988).

[11] K. Fujita, T. Yamamoto, T. Takebe, T. Watanabe. Jpn. J. Appl. Phys., 32, L978 (1993).

[12] D.H. Rich, K. Rammohan, Y. Tang, H.T. Lin. J. Vac. Sci. Technol. B Microelectron. Nanometer Struct. Process. Meas. Phenom., 13, 1766 (1995).

[13] M.M. Lira, J.L. Alvarado, M.L. Lípez, M.A. Vidal. Superf. Vacío, 32 (2000).

[14] D.G. Vasil'ev, V.P. Evtikhiev, V.E. Tokranov, I.V. Kudryashov, V.P. Kochereshko. Phys. Solid State, 40, 787 (1998).

[15] K. Mochizuki, S. Goto, H. Kakibayashi, C. Kusano. Jpn. J. Appl. Phys., 29, L1046 (1990).

[16] P.R. Hageman, J. te Nijenhuis, M.J. Anders, L.J. Giling. J. Cryst. Growth, 170, 270 (1997).

[17] S. Orsila, A. Tukiainen, P. Uusimaa, J. Dekker, T. Leinonen, M. Pessa. J. Cryst. Growth, 227-228, 249 (2001).

[18] R.C. Tu, Y.K. SuS. T. Chou. J. Appl. Phys., 84, 6877 (1998).

[19] P.V. Seredin, V.E. Ternovaya, A.V. Glotov, A.S. Len'shin, I.N. Arsent'ev, D.A. Vinokurov, I.S. Tarasov, H. Leiste, T. Prutskij. Phys. Solid State, 55, 2161 (2013).

[20] P.V. Seredin, A.V. Glotov, E.P. Domashevskaya, I.N. Arsentyev, D.A. Vinokurov, I.S. Tarasov. J. Phys. B: Condens. Matter, 405, 2694 (2010).

[21] W. Hayes, R. Loudon. Scattaring of Light by Crystals (John Wiley \& Sons, N.Y., 1978).

[22] P.V. Seredin, E.P. Domashevskaya, V.E. Ternovaya, I.N. Arsent'ev, D.A. Vinokurov, I.S. Tarasov, T. Prutskij. Phys. Solid State, 55, 2169 (2013).

[23] A.P.V. Seredin, A.V. Glotov, A.S. Lenshin, I.N. Arsentyev, D.A. Vinokurov, T. Prutskij, H. Leiste, M. Rinke. Semiconductors, 48, 21 (2014).

[24] P.V. Seredin, A.V. Glotov, E.P. Domashevskaya, I.N. Arsentyev, D.A. Vinokurov, I.S. Tarasov, I.A. Zhurbina. Semiconductors, 44, 184 (2010).

[25] И.Н. Арсентьев, Е.С. Калевич, С.Г. Конников, В.М. Лантратов, Т.Б. Попова, В.К. Тибилов, В.П. Улин. Автометрия, 74 (1981).

Редактор А.Н. Смирнов 


\section{Influence of misorientation \\ on composition, structural \\ and photoluminescence properties of epitaxial layers grown on GaAs(100)}

P.V. Seredin ${ }^{1}$, A.S. Lenshin ${ }^{1}$, A.V. Fedyukin ${ }^{1}$, I.N. Arsentyev ${ }^{2}$, A.V. Zhabotinsky', D.N. Nikolaev ${ }^{2}$, H. Leiste ${ }^{3}$, M. Rinke ${ }^{3}$

${ }^{1}$ Voronezh State University, 394006 Voronezh, Russia

2 loffe Institute, 194021 St. Petersburg, Russia

${ }^{3}$ Instituto de Ciencias,

Benemérita Universidad Autónoma de Puebla, 72050 Puebla, Pue., Mexico

Abstract The influence of substrate misorientation and pretreatment on the structural and optical characteristics of GaAs/GaAs(100) homoepitaxial structures, grown by MOCVD has been investigated. On the basis of complex structural and spectroscopic characteristics it was demonstrated, that the increase of the substrate's deviation from the precise orientation of up to $4^{\circ}$ toward [110] leads to a stepwise growth of the GaAs films at the initial stage, and a further increase of misorientation up to $10^{\circ}$ to the [110] leads to an increase in the defects in the structure of the epitaxial film. In this case, the samples of homoepitaxial structures, grown by MOCVQ on the $\operatorname{GaAs}(100)$ substrates with the $4^{\circ}$ disordering toward [110] have maximum of photoluminescence. Pre-polishing of the GaAs substrate (the remove of the oxide layer) also leads to the emission's increasing compared to the unpolished same type substrates, while for samples grown on substrates with $4^{\circ}$ disordering such increase of the photoluminescence in the output is the value of $\sim 30 \%$. 